\title{
Spots on EK Draconis
}

\section{Active longitudes and cycles from long-term photometry ${ }^{\star}$}

\author{
S. P. Järvinen ${ }^{1,2}$, S. V. Berdyugina ${ }^{2,3}$, and K. G. Strassmeier ${ }^{1}$ \\ 1 Astrophysikalisches Institut Potsdam, An der Sternwarte 16, 14482 Potsdam, Germany \\ e-mail: [sjarvinen;kstrassmeier]@aip.de \\ 2 Astronomy Division, PO Box 3000, 90014 University of Oulu, Finland \\ 3 Institute of Astronomy, ETH Zentrum, 8092 Zürich, Switzerland \\ e-mail: sveta@astro.phys.ethz.ch
}

Received 25 April 2005 / Accepted 30 May 2005

\begin{abstract}
We analyse photometric observations of the young active star EK Dra, altogether about 21 years. Similar to the ZAMS stars LQ Hya and AB Dor, EK Dra shows long-lived, non-axisymmetric spot distribution with active longitudes on opposite hemispheres. At least two activity cycle can be found from the data. The first cycle originates from repeated switches of the activity between two active longitudes in about (2-2.25)-year intervals, resulting in a cycle of about (4-4.5) years. The second cycle is of the order of 10.5 years and comes from migration of the active longitudes. Our data cover two consecutive cycles. The periodicity is also present in the maximum, the mean and the minimum stellar brightness. Additionally, there is a long-term trend. If we combine our data with the Sonneberg plate measurements, we can conclude that the overall brightness of EK Dra has been continuously decreasing at least for the last 45 years. A comparison with current sunspot activity reveals many similarities between the Sun and EK Dra.
\end{abstract}

Key words. stars: activity - stars: starspots

\section{Introduction}

EK Dra (HD 129333) is an active, G1.5 V star in the northern sky. The rotation period of the star of 2.6 days confirms its rapid rotation of about 10 times faster than the Sun. It was originally found during the survey of Ca II $\mathrm{H}$ and $\mathrm{K}$ emission in solar neighbourhood stars by Vaughan \& Preston (1980). It is identified as a member of the Pleiades moving group, which is also known as the Local Association (Montes et al. 2001). Its distance has been measured to be $33.9 \mathrm{pc}$ by HIPPARCOS which, together with the brightest state so far observed for EK Dra, i.e. $V=7{ }^{\mathrm{m}} \cdot 51$, leads to its absolute magnitude of $M_{V}=4.86$ and thus verifies its spectral classification.

The light variations of EK Dra were first discovered by Chugainov et al. (1991) and later independently by Dorren \& Guinan (1994). The long-term photometric period is measured to be 2.605 days from a subset of the present data (Strassmeier \& Rice 1998). Earlier photometric data suggested the existence of long-term brightness variations with periods of approximately 12-14 years (Dorren \& Guinan 1994; Dorren et al. 1995). Additional data allowed Messina \& Guinan (2002) to identify a 9.2 year cycle with a long-term trend of more than

* Table 2 is only available in electronic form at the CDS via anonymous ftp to cdsarc.u-strasbg.fr $(130.79 .128 .5)$ or via http://cdsweb.u-strasbg.fr/cgi-bin/qcat?J/A+A/440/735
30 years. The long-term trend may be even closer to 40 years because the study of Sonneberg Sky-Patrol plates (Fröhlich et al. 2002) showed that the star's $B$ magnitude declined during the time span of these plates of 35 years and continued to decline in subsequent photoelectric photometry.

Strassmeier \& Rice (1998) carried out the first, and so far only, Doppler-imaging analysis of EK Dra and found a single dominating cool spot located consistently at a latitude of $\approx 70-80^{\circ}$, thus, far in excess of where our Sun shows spots. The existence of such a very high-latitude spot is only qualitatively in agreement with the flux-tube transportation model of Schüssler et al. (1996) and thus requires an additional physical process that moves toroidal flux tubes to very high latitudes. Strassmeier \& Rice (1998) also noticed that there are two spot groups separated by about $180^{\circ}$ in longitude, which could not be artifacts due to a wrong rotational period, because the rotation period would be only a half of the actual 2.6-day rotation period, according to light curve morphology and asymmetry.

EK Dra has an ultraviolet spectrum with strong and variable chromospheric emission lines (Dorren \& Guinan 1994), frequent low-level flaring of the transition-region lines C IV and Si IV (Saar \& Bookbinder 1998), strong non-thermal radio emission (Güdel et al. 1995a) and rotationally modulated X-ray emission (Güdel et al. 1995b) from a 7-18 MK hot 
coronal plasma with a X-ray luminosity of $\log L_{\mathrm{X}} \approx$ $29.8 \mathrm{erg} \mathrm{s}^{-1}$ (Güdel et al. 1997a,b), which is about 300 times stronger than for the Sun (Dorren \& Guinan 1992).

EK Dra is the brightest young solar analog. It shows strong magnetic activity and it still represents the best stellar approximation of the young solar atmosphere, although its atmospheric structure may not be directly comparable to the present Sun. Earlier photometric studies of two other young ZAMS stars, LQ Hya (Berdyugina et al. 2002) and AB Dor (Järvinen et al. 2005), showed that both of them appear to have two active longitudes about $180^{\circ}$ apart dominating the activity of the stars. Migration of the active longitudes suggested the presence of weak differential rotation on their surfaces, which has been confirmed by Doppler imaging (Donati \& Collier Cameron 1997, Kövári et al. 2004).

Comparing LQ Hya with the Sun, Berdyugina et al. (2002) concluded that the activity of the young Sun could also consist of two dynamo modes, axi- and non-axisymmetric. During solar evolution, as the Sun lost angular momentum, the power of the non-axisymmetric mode should have significantly decreased. A recent study of sunspot activity has, however, revealed that the non-axisymmetric mode is still excited in the present Sun (Berdyugina \& Usoskin 2003). Moreover, the solar active longitudes associated with such a mode switch their dominant activity with a 3.7 year flip-flop cycle, which is $1 / 3$ of the 11-year cycle, similar to LQ Hya.

The above results encouraged us to collect all available photometric data of EK Dra and analyse them to establish whether the star exhibits active longitudes and cyclic behaviour in spot patterns and overall spot activity. The observations and analysis methods are described in Sect. 2. The obtained results are presented in Sect. 3. In Sect. 4, we discuss the activity patterns of EK Dra and compare them with those of the Sun and other active stars, and we summarise our findings in Sect. 5.

\section{Data and analysis}

\subsection{Observations}

In order to search for long-lived spot structures and activity cycles, we collected all available photometric records from the literature, added new observations, and compiled a dataset covering 21 years from the beginning of 1984 to the end of 2004. Some of the previously published data were in the Strömgren uvby system (Lockwood et al. 1997), but were transformed into Johnson $V$ magnitudes according to Olsen (1983). The rest of the already published data were in the standard Johnson $U B V R I$ system.

The new data presented in this paper started in 1997/1998 and were taken with Amadeus, one of the two $0.75 \mathrm{~m}$ automatic photoelectric telescopes (APT) of the University of Vienna at the Fairborn Observatory in southern Arizona. Amadeus is optimised for red wavelengths with an EMI-9828 tube and Johnson-Cousins $V\left(R I_{\mathrm{C}}\right)$ filters (for more details see Strassmeier et al. 1997b). Measurements were made differentially between the variable, a comparison star, a check star and the sky position. The data reduction is also automatic and is described in Strassmeier et al. (1997a) and Granzer et al. (2001).
Table 1. Sources of the photometric data.

\begin{tabular}{lrlrl}
\hline \hline $\begin{array}{l}\text { JD range } \\
\text { (JD 2 400 000+) }\end{array}$ & $N$ & Source & C & Ck \\
\hline $45781-50253$ & 370 & Lockwood et al. (1997) & 1 & 2 \\
$49398-50263$ & 295 & Strassmeier et al. (1997a) & 2 & 3 \\
$50425-50569$ & 271 & Strassmeier et al. (1999) & 2 & 3 \\
$50807-53168$ & 1521 & This paper (Amadeus APT) & 2 & 3 \\
$51645-52071$ & 16 & Alekseev (2003) & 2 & 3 \\
$52332-52392$ & 11 & Alekseev (2004) & 2 & 3 \\
$52740-52773$ & 11 & Alekseev (2005) & 2 & 3 \\
\hline
\end{tabular}

Comparison (C) and Check (Ck) stars:

$1=$ HD 131330; $2=$ HD 129390; $3=$ HD 129798.

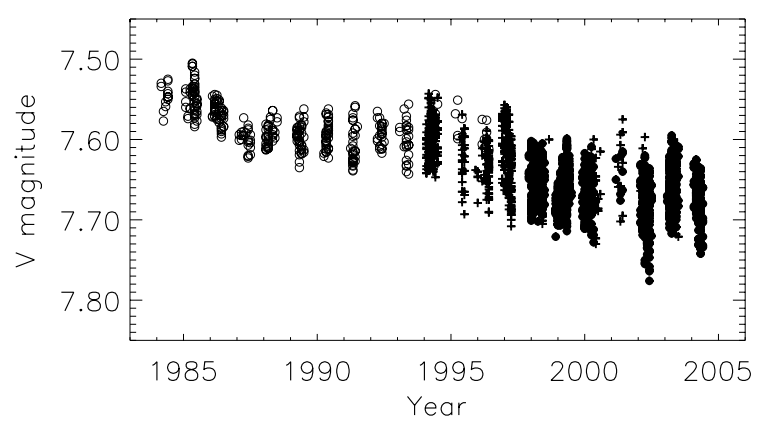

Fig. 1. The photometric data analysed in this paper. Open circles denote Strömgren $y$-band data which is transformed into Johnson $V$ magnitudes, + symbols denote $V$-band photometry and filled circles represent the new data.

The sources of the published observations along with the comparison (C) and check (Ck) stars are given in Table 1. The unpublished Amadeus data are given in Table 2, which is available at the CDS and contains the following information. Column 1 is the name of the source, Col. 2 gives the time of the observations, Col. 3 gives the differential $V$ magnitude and Col. 4 gives the standard error. The observations used are presented in Fig. 1. The errors of the observations were typically 0.006.

The entire 21 years of data were divided into 60 sets, so that most of the sets had a good phase coverage over the rotational period and each set represented a light curve stable for a given time interval. The observations were phased with the ephemeris of:

$T=2445781.859+2.606 E$.

\subsection{Light curve inversions}

In order to determine locations of spots on the stellar surface, we performed inversions of the observed light curves into stellar images. The approach is based on the Occamian inversion technique (Berdyugina 1998; Berdyugina et al. 2002). The model assumes that, due to the low spatial resolution, the local intensity of the stellar surface always contains contributions from the photosphere and from cool spots weighted by the fraction of the surface covered by spots i.e. the spot filling factor. The inversion of a light curve results in a distribution 
of the spot filling factor over the visible stellar surface. Since a light curve represents a one-dimensional time series the resulting stellar image contains information mostly in the direction of rotation, i.e. in longitude, while spot size and locations in latitude remain comparably ill determined. However, because of projection effects and limb darkening, the inverse technique can recover more structure than is obvious at first glance from the shape of the light curve and is very useful for determining longitudes of spot concentrations. Although this approach is less informative than the Doppler imaging technique, the analysis of long series of photometric observations allows us to recover longitudinal spot patterns and study their long-term evolution.

For EK Dra, values of the photospheric and spot temperatures were assumed to be equal to $5800 \mathrm{~K}$ and $4500 \mathrm{~K}$, respectively. The temperature of the photosphere corresponds to the effective temperature of a G2 star, while spots were assumed to be cooler by $1300 \mathrm{~K}$, which is typical for solar-type stars. The Doppler imaging study by Strassmeier \& Rice (1998) recovered a spot temperature in 1995 of $\Delta T \approx 1200 \mathrm{~K}$ from 12 major spectral lines. The photometric study of Alekseev (2003) indicated that spots may even be $2200 \mathrm{~K}$ cooler than the photosphere, a result that is quite similar to the result of O'Neil et al. (2004) based on TiO-band observations. Because we were interested only in spot longitudes, possible errors in the temperature values were not important. For the inversions, the stellar surface was divided into a grid of $10^{\circ} \times 10^{\circ}$, and the spot filling factor was determined for each grid pixel. The recovered stellar images and fits to the observed light curves are shown in Fig. 2.

\section{Results}

\subsection{Active longitudes}

Spot longitudes at the locations of the maximum spot filling factors were recovered from the images. Two filling factor maxima were seen in most of the cases, which indicated two spot concentrations on the stellar surface, with one generally being larger, called the primary spot concentrations. If only one spot is present, which is sometimes the case when light-curves have a very broad minimum, it is considered the primary concentration. The time dependence of the spot phases is shown in Fig. 3. In all cases the exact phases of the spots are uncertain due to the broad light-curve minima and/or the incompleteness of the light curve. The errors in spot phases are determined from the full width at half maxima of the fitted shapes of the light-curve minima and are also plotted in Fig. 3. The numerical values are given in Table 3.

We interpret the two spot concentrations as two active longitudes about $180^{\circ}(\Delta \varphi=0.5)$ apart. This is visualised in Fig. 4 where a clear two-peaked distribution of the spots with respect to the one of the traced active longitudes is seen. The sinusoidal drift of both active longitudes is depicted in Fig. 3. In 1984-1987 spots migrated to later phases, then in 1987-1992 to earlier phases, in 1992-1997 to later phases, in 1997-2002 to earlier phases, and again after 2002 they seem to migrate again to later phases. Because phases were calculated with a fixed value for the photometric (=rotational) period, the drift indicates changes of the spot rotation period on a time scale of about 10 years.

\subsection{Differential rotation and solar-type cycle}

Messina \& Guinan (2003) have reported that EK Dra shows solar-type surface differential rotation, i.e. with faster rotation at lower latitudes. However, the strength of the differential rotation of EK Dra is still unknown. The shape of the migration paths of the active longitudes implies continuous variations of the spot rotation period. In the presence of solar-type differential rotation, this would suggest that the emergence latitude of the spots changes. Spots drift from higher latitudes towards lower latitudes until 1992 when they appear again at higher latitudes and then start a new drift towards lower latitudes until 2002 when they appear again at higher latitudes. In the Sun, the mean latitude changes are accompanied by total sunspot area variations during the 11-year sunspot cycle. A similar behaviour is observed for EK Dra. As seen from Figs. 1 and 3, the overall activity level of EK Dra and the spot rotation period vary on the same time scale, approximately 10.5 years, which suggests the presence of a sunspot-like cycle on EK Dra. A very similar behaviour was detected on $\mathrm{AB}$ Dor with a cycle period of 20 years (Järvinen et al. 2005).

Dorren \& Guinan (1994) suggested that EK Dra has an activity cycle of 12 years based on photometry from 1982-1994. This result was questioned by Saar \& Brandenburg (1999). Recently, Messina \& Guinan $(2002,2003)$ reported a primary period of 9.2 years and an additional long-term trend, which may have a cycle of more than 30 years if it is periodical. Because the overall brightness of EK Dra decreases during the observations (Fig. 1), which cover 21 years, and the longterm plate magnitudes obtained by Fröhlich et al. (2002) also showed a continuous decrease of the $B$ magnitude for an additional 25 years before the first data points of this study, we can conclude that the overall brightness of EK Dra has been decreasing at least for the last 45 years.

The length of the cycle on EK Dra can be estimated using the photometric data presented in Fig. 1. Using a second order Fourier fit to the brightness variations we find the cycle length to be about 10.5 years with an additional long-term trend. Fits to the maximum, mean and minimum magnitudes in Fig. 5 also show a cycle of about 10.5 years, plus the long-term trend. These cycles are further confirmed by a spectral analysis using the Lomb method for unevenly sampled data (Press et al. 1992). The turnover points of the migration paths in 1992 and in 2002/2003 may indicate the beginning of a new cycle, as we know it from the Sun (Berdyugina \& Usoskin 2003).

\subsection{Flip-flop cycle}

When the dominant spot concentration switches to another active longitude we may observe a so-called flip-flop. This effect was found to be periodic in RS CVn-type stars, FK Com, the young solar analogs LQ Hya and AB Dor and the Sun (Berdyugina \& Tuominen 1998; Korhonen et al. 2002; 


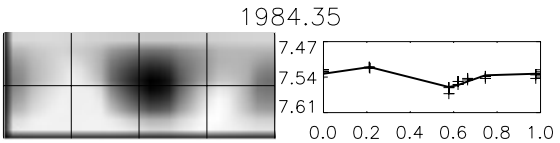

1985.27

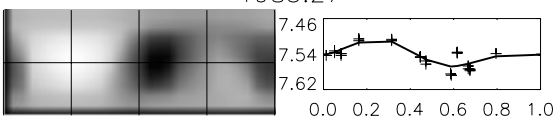

1985.45
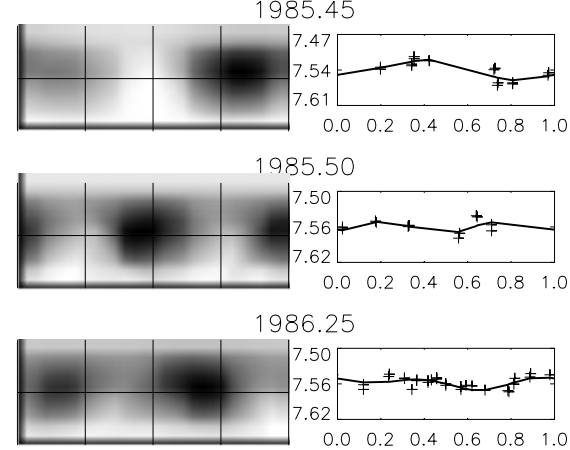

1986.45

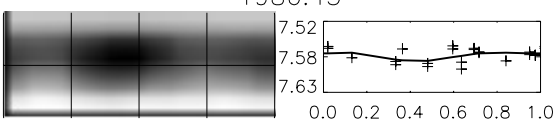

1987.30

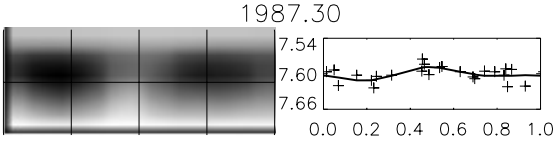

1988.31

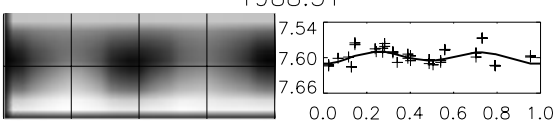

1989.41

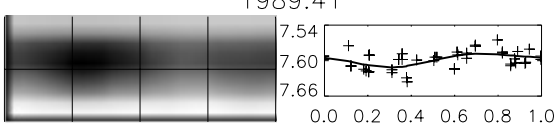

1990.40
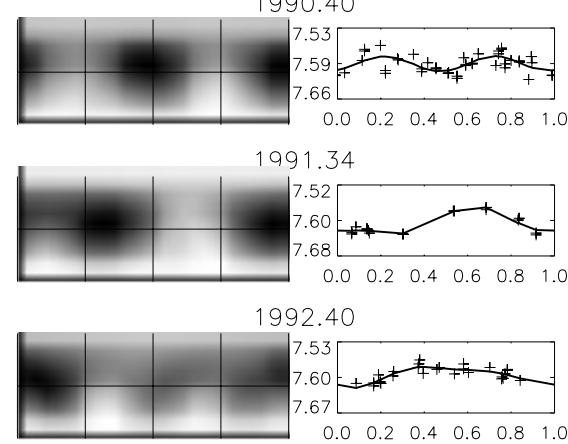

1992.40

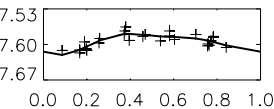

1993.25
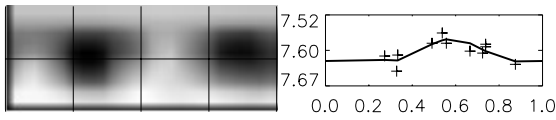

1993.42
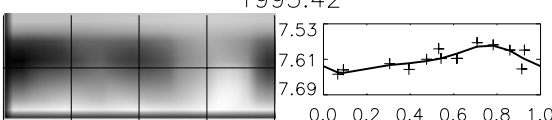

Fig. 2. The light-curve inversion results. Images are shown in a gray scale with the spot filling factor being larger in darker regions. The coordinate grid in the images indicates positions of the equator and four longitudes separated by $90^{\circ}$. Observed and calculated $V$-band light curves are presented by crosses and lines, respectively. Note the changing vertical scale in the light curve plots.
1997.13
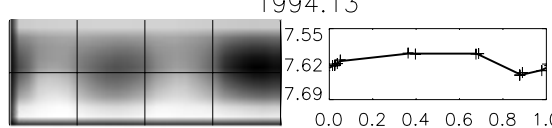

1994.16

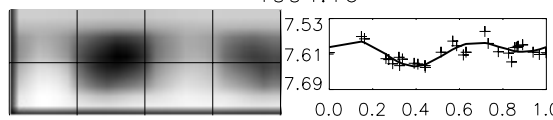

1994.27
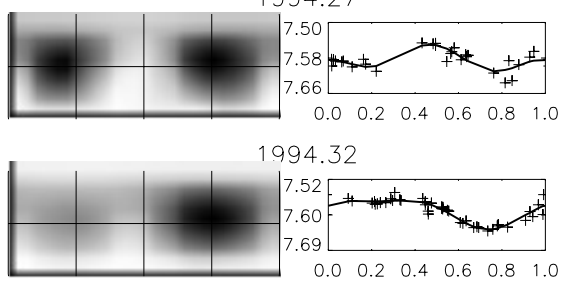

1994.37
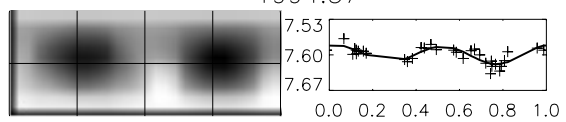

1994.41

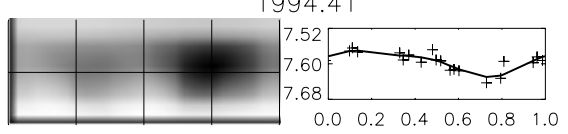

1994.45
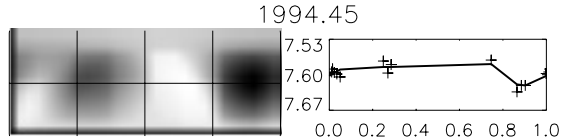

994.52

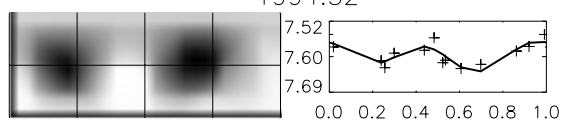

1995.42

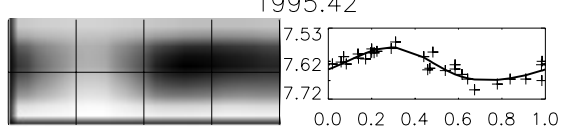

1996.20
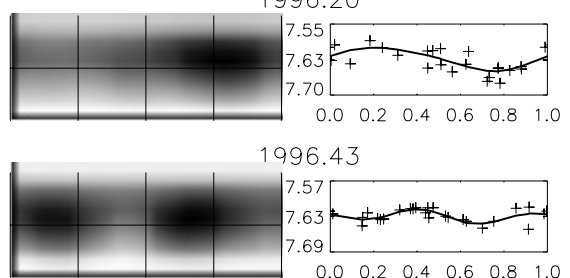

996.43
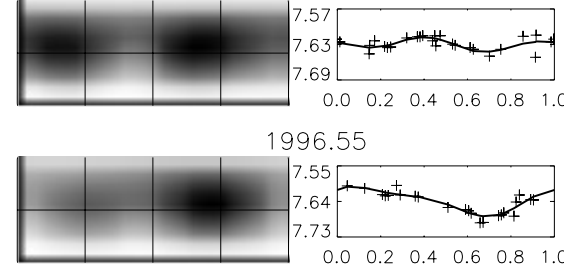

996.55

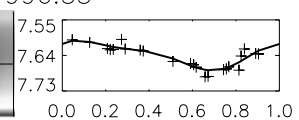

1997.01
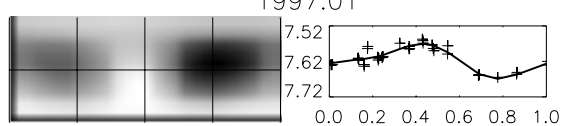

1997.09
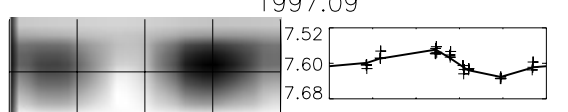

1997.19

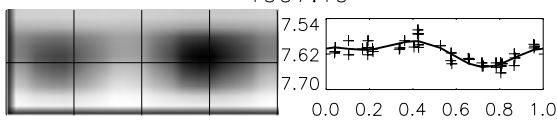

1997.28
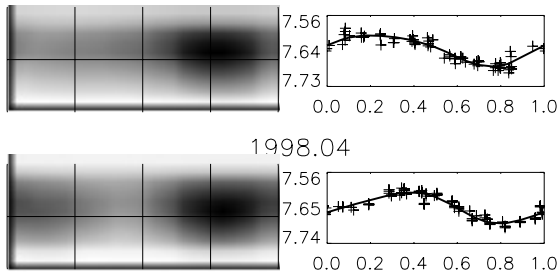

1998.17

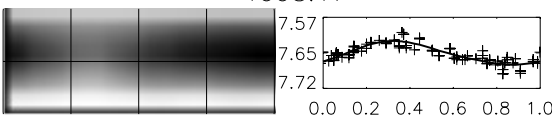

1998.27

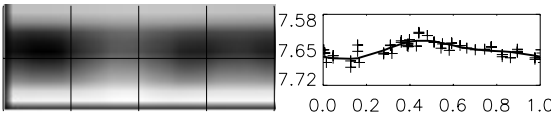

1998.33

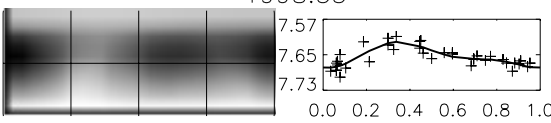

1998.42

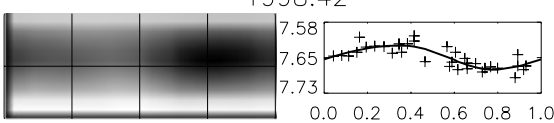

1998.48

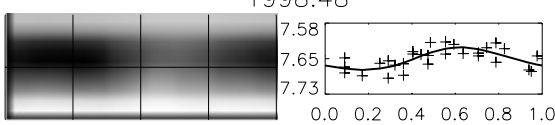

1998.92

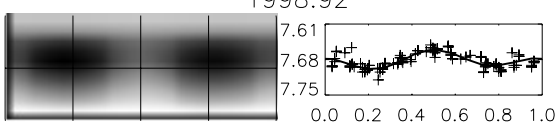

1999.15

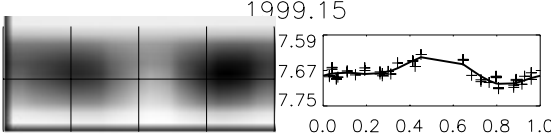

1999.22

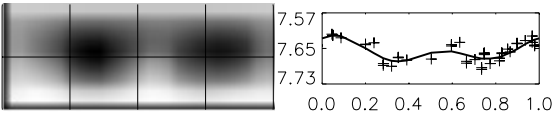

1999.29

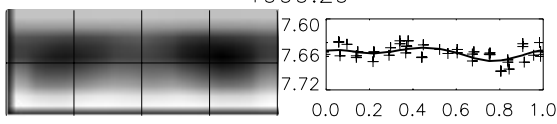

1999.34

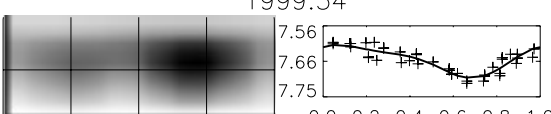




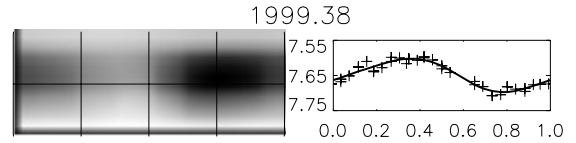

1999.45

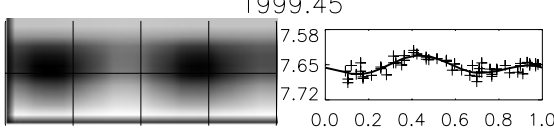

2000.00

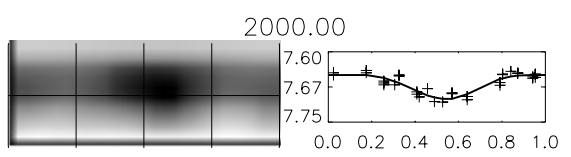

2000.05
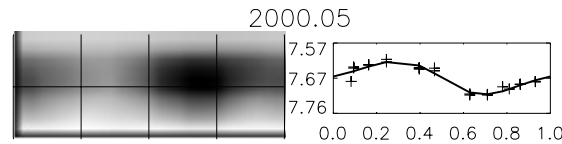

2000.11
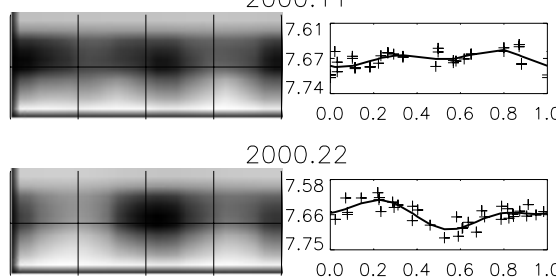

2000.22

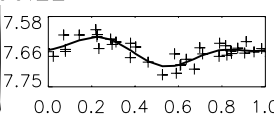

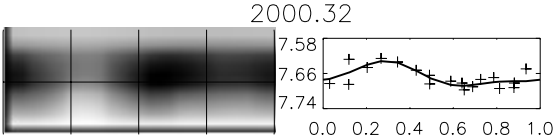

2000.39

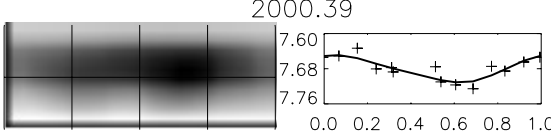

2001.34

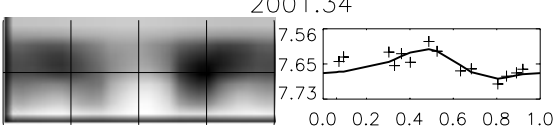

2002.22
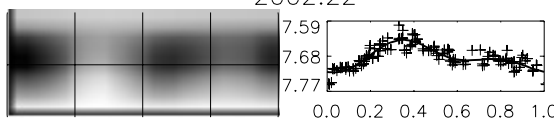

2002.38
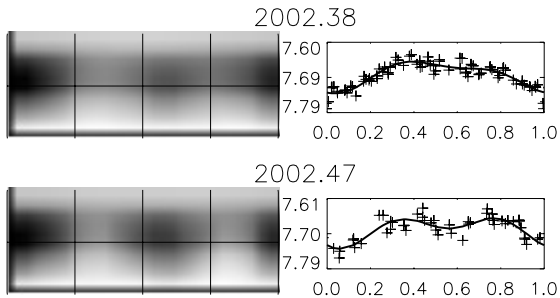

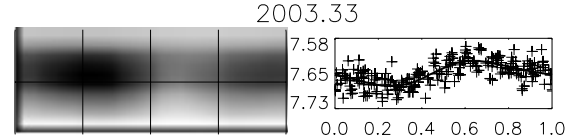

2004.18

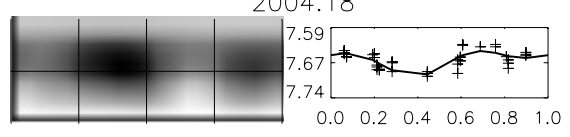

2004.25

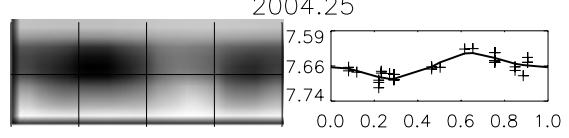

2004.32

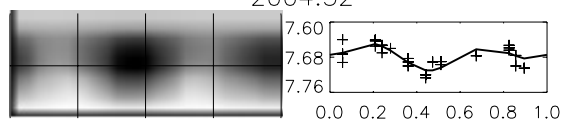

2004.35
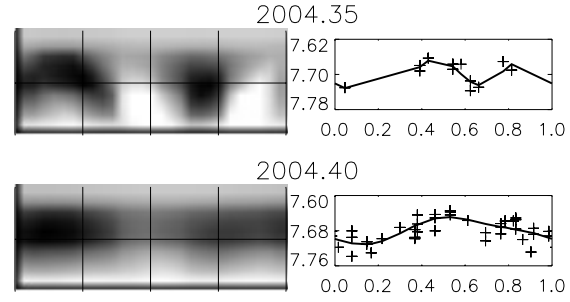

Fig. 2. continued.

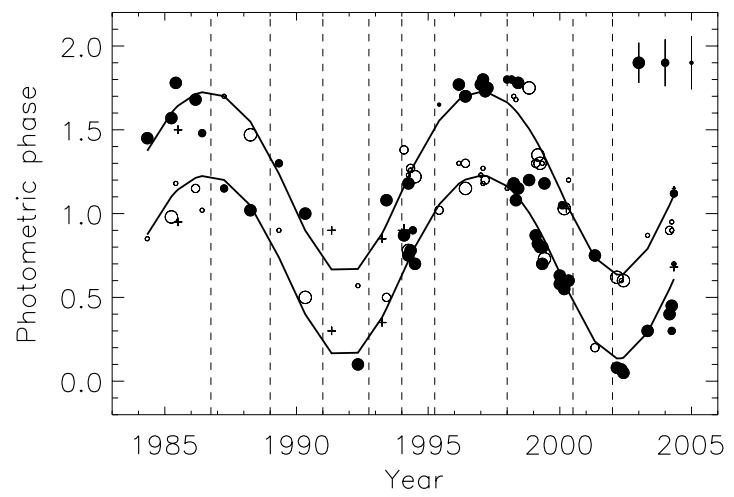

Fig. 3. Phases of the spots recovered on the surface of EK Dra using the light curve inversion technique. Filled circles denote primary spots, open circles secondary spots, and + symbol is used when it is not possible to say which spot is the primary one. Two active longitudes are traced by solid lines. Different symbol sizes correspond to different error bars shown in the top right corner of the plot.

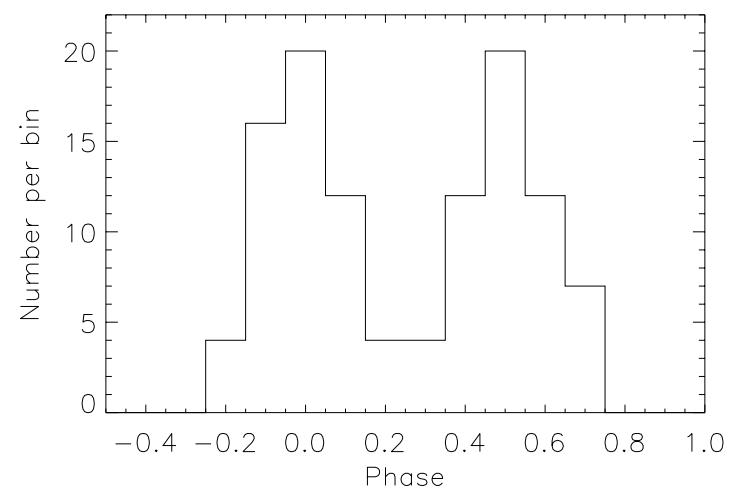

Fig. 4. Histogram of the recovered spot locations with respect to the one of the active longitudes traced in Fig. 3. Two peaks clearly show up at phases 0.0 and 0.5 .

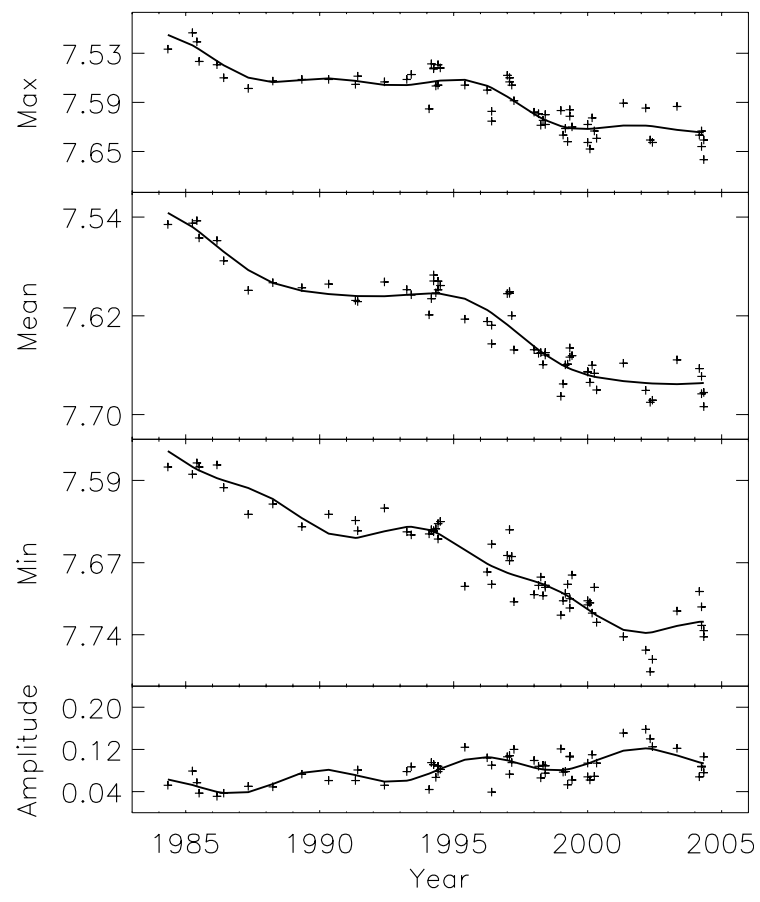

Fig. 5. Maximum, mean, minimum and peak-to-peak amplitude magnitudes measured from individual light curves (crosses). Double harmonic fits to the variations (solid lines) reveal the cycles in the data. The maximum, mean and minimum magnitudes decrease while the peak-to-peak amplitude increases with a long-term trend. The maximum, mean and minimum magnitudes vary also with an about 10.5 years cycle. Furthermore, the amplitude varies with a $6.5-\mathrm{yr}$ period.

Berdyugina et al. 2002; Järvinen et al. 2005; Berdyugina \& Usoskin 2003). Since these stars show cyclic flip-flops, we looked for such a cycle in the activity of EK Dra. 
Table 3. Spot parameters: JD is the Julian date -2400000 at the beginning and at the end of data set and $\varphi$ is the recovered phase of the active region 1 and 2, respectively. Com is a comment column where 1 means "incomplete light curve" and 2 "broad light curve".

\begin{tabular}{|c|c|c|c|c|c|c|c|c|c|c|c|c|c|}
\hline Set & Year & JD (beg) & JD (end) & $\varphi_{1}$ & $\varphi_{2}$ & Com & Set & Year & JD (beg) & JD (end) & $\varphi_{1}$ & $\varphi_{2}$ & Com \\
\hline 1 & 1984.35 & 45781 & 45874 & 1.45 & 0.85 & 1 & 31 & 1997.28 & 50531 & 50568 & 1.75 & - & 2 \\
\hline 2 & 1985.27 & 46104 & 46223 & 1.57 & 0.98 & & 32 & 1998.04 & 50807 & 50848 & 1.80 & 1.15 & \\
\hline 3 & 1985.45 & 46224 & 46239 & 1.78 & 1.18 & 1 & 33 & 1998.17 & 50850 & 50901 & 1.80 & - & 2 \\
\hline 4 & 1985.50 & 46244 & 46252 & 1.50 & 0.95 & 1 & 34 & 1998.27 & 50903 & 50922 & 1.18 & 1.70 & \\
\hline 5 & 1986.25 & 46488 & 46554 & 1.68 & 1.15 & & 35 & 1998.33 & 50923 & 50949 & 1.08 & 1.68 & \\
\hline 6 & 1986.45 & 46567 & 46625 & 1.48 & 1.02 & & 36 & 1998.42 & 50953 & 50979 & 1.78 & - & 2 \\
\hline 7 & 1987.30 & 46829 & 46983 & 1.15 & 1.70 & & 37 & 1998.48 & 50980 & 50998 & 0.15 & - & \\
\hline 8 & 1988.31 & 47199 & 47348 & 1.02 & 1.47 & & 38 & 1998.92 & 51076 & 51221 & 1.20 & 1.75 & \\
\hline 9 & 1989.41 & 47624 & 47725 & 1.30 & 0.90 & 2 & 39 & 1999.15 & 51222 & 51247 & 0.87 & 1.30 & \\
\hline 10 & 1990.40 & 48008 & 48066 & 1.00 & 0.50 & & 40 & 1999.22 & 51248 & 51268 & 0.82 & 1.35 & \\
\hline 11 & 1991.34 & 48369 & 48390 & 0.30 & 0.90 & 1,2 & 41 & 1999.29 & 51274 & 51295 & 0.80 & 1.30 & \\
\hline 12 & 1992.40 & 48732 & 48806 & 0.10 & 0.57 & & 42 & 1999.34 & 51296 & 51311 & 0.70 & 1.30 & \\
\hline 13 & 1993.25 & 49030 & 49128 & 0.35 & 0.85 & 1 & 43 & 1999.38 & 51312 & 51325 & 0.80 & - & 2 \\
\hline 14 & 1993.42 & 49129 & 49156 & 1.08 & 0.50 & 2 & 44 & 1999.45 & 51326 & 51361 & 1.18 & 0.73 & \\
\hline 15 & 1994.13 & 49398 & 49406 & 0.90 & - & 1 & 45 & 2000.00 & 51534 & 51555 & 0.58 & - & 2 \\
\hline 16 & 1994.16 & 49410 & 49429 & 0.87 & 1.38 & 1 & 46 & 2000.05 & 51556 & 51572 & 0.63 & - & 2 \\
\hline 17 & 1994.27 & 49441 & 49458 & 1.18 & 0.78 & & 47 & 2000.11 & 51573 & 51596 & 1.05 & 0.58 & \\
\hline 18 & 1994.32 & 49462 & 49479 & 0.75 & 1.23 & & 48 & 2000.22 & 51601 & 51649 & 0.55 & 1.03 & \\
\hline 19 & 1994.37 & 49481 & 49496 & 0.78 & 1.27 & 1 & 49 & 2000.32 & 51650 & 51672 & 0.60 & 1.03 & \\
\hline 20 & 1994.41 & 49501 & 49509 & 0.78 & 1.26 & 2 & 50 & 2000.39 & 51674 & 51699 & 0.60 & 0.20 & 2 \\
\hline 21 & 1994.45 & 49510 & 49526 & 0.90 & - & 1 & 51 & 2001.34 & 51992 & 52078 & 0.20 & 0.75 & \\
\hline 22 & 1994.52 & 49536 & 49548 & 0.70 & 1.22 & 1 & 52 & 2002.22 & 52326 & 52392 & 0.08 & 0.62 & \\
\hline 23 & 1995.42 & 49832 & 49909 & 1.65 & 1.02 & 2 & 53 & 2002.38 & 52397 & 52431 & 0.07 & 0.60 & \\
\hline 24 & 1996.20 & 50082 & 50229 & 1.77 & 1.30 & 2 & 54 & 2002.47 & 52432 & 52463 & 0.05 & 0.60 & \\
\hline 25 & 1996.43 & 50230 & 50249 & 1.70 & 1.15 & & 55 & 2003.33 & 52691 & 52830 & 0.30 & 0.87 & \\
\hline 26 & 1996.55 & 50250 & 50263 & 1.70 & 1.30 & 2 & 56 & 2004.18 & 53052 & 53090 & 0.40 & 0.90 & \\
\hline 27 & 1997.01 & 50434 & 50468 & 1.77 & 1.23 & 2 & 57 & 2004.25 & 53091 & 53115 & 0.30 & 0.90 & \\
\hline 28 & 1997.09 & 50473 & 50489 & 1.80 & 1.18 & 1 & 58 & 2004.32 & 53116 & 53128 & 0.45 & 0.95 & \\
\hline 29 & 1997.13 & 50490 & 50504 & 1.77 & 1.27 & 2 & 59 & 2004.35 & 53129 & 53135 & 0.15 & 0.68 & 1 \\
\hline 30 & 1997.19 & 50510 & 50529 & 1.73 & 1.20 & & 60 & 2004.40 & 53137 & 53168 & 0.12 & 0.70 & \\
\hline
\end{tabular}

In Fig. 3 primary spots (filled circles) jump rather regularly from one active longitude to another, indicating the presence of the flip-flop effect. Such moments are emphasised by vertical dashed lines. On average the flip-flops happen with about 2-2.25 year intervals and, thus, suggest a flip-flop cycle of about $4-4.5$ years.

\section{Discussion}

The two spot groups separated by about $180^{\circ}$ on EK Dra had already been detected from earlier light curves by Scheible \& Guinan (1994) and were discussed by Strassmeier \& Rice (1998). Although Strassmeier \& Rice (1998) studied light curve variability of EK Dra over seven months from December 1996 to July 1997, and detected changes and slight shifts in phases of the spots, the limited data set did not allow the recovery of the migration of the active longitudes, which becomes evident on a longer time-scale.

The only Doppler image of EK Dra by Strassmeier \& Rice (1998) can be compared with our map from set 22 (Table 3). If our observations are phased with the same ephemeris as used by Strassmeier \& Rice, the light curve minimum is centred at $\varphi=0.13$ (corresponding to longitude $l=47^{\circ}$ ) while Strassmeier \& Rice report the large high-latitude feature at longitude $l=45^{\circ}$.
The migration pattern of the active longitudes revealed by us on EK Dra is very similar to that observed on the Sun over the 11-year cycle (Berdyugina \& Usoskin 2003). The two turnover times in Fig. 3 support a repeating 10.5-yr cycle on EK Dra. As discussed previously by Berdyugina (2004) and Berdyugina \& Järvinen (2005), together with LQ Hya, AB Dor and the Sun, the G1.5V-star EK Dra belongs to the group of Sun-like stars that are distinguished by the following specific activity patterns:

(1) cyclic variations of the mean activity level;

(2) differential rotation;

(3) migrating active longitudes separated by $180^{\circ}$; and

(4) a flip-flop cycle.

Berdyugina et al. (2002) suggested that this implies the coexistence of both axisymmetric and non-axisymmetric dynamo modes. The relative strength of the modes can vary on the evolutionary time scale, with a non-axisymmetric mode being stronger in younger stars, which rotate more rapidly. The role of the rapid rotation is emphasised by the fact that evolved binary components (RS CVn type stars) and apparently single rapidly rotating giants (FK Com type stars) also show similar phenomena (Berdyugina \& Tuominen 1998; Korhonen et al. 2002). A theoretical model of these phenomena is being developed, and the observational constraints for such a model 
are of great importance (see, e.g. Moss 2004, 2005; Fluri \& Berdyugina 2004; Elstner \& Korhonen 2005).

\section{Summary}

In this paper, we presented photometric data of the young active star EK Dra, and analysed them with the technique of lightcurve inversion. Such an approach reveals the longitudinal spot distribution and is good enough to study active longitudes and their evolution. The following results were obtained:

- Spots on the surface of EK Dra are grouped around two active longitudes separated on average by $180^{\circ}$.

- The total spot area varies on a time scale longer than 45-yr with an additional periodic variation of about 10.5 years. This period is also seen in the migration paths of the active longitudes.

- Periodic switches of the dominant activity between the active longitudes results in a flip-flop cycle of about 4-4.5 years.

- Magnetic activity of EK Dra is similar to that observed in the other young ZAMS stars LQ Hya and AB Dor, the single giant FK Com, some components of the RS CVn-type binaries, and the Sun.

Acknowledgements. We acknowledge I. Yu. Alekseev for providing his observations. S. P. Järvinen acknowledges support by the Deutsche Forschungsgemeinschaft, grant KO 2320/1. S. V. Berdyugina aknowledges support by the Academy of Finland, grant 43039.

\section{References}

Alekseev, I. Yu. 2003, ARep, 47, 430

Alekseev, I. Yu. 2004, Sol. Phys., 224, 187

Alekseev, I. Yu. 2005, Astrophys., 48, 20

Berdyugina, S. V. 1998, A\&A, 338, 97

Berdyugina, S. V. 2004, Sol. Phys., 224, 123

Berdyugina, S. V., \& Järvinen, S. P. 2005, AN, 326, 283

Berdyugina, S. V., \& Tuominen I. 1998, A\&A, 336, L25

Berdyugina, S. V., \& Usoskin, I. G. 2003, A\&A, 405, 1121

Berdyugina, S. V., Pelt, J., \& Tuominen, I. 2002, A\&A, 394, 505

Chugainov, P. F., Lovkaya, M. N., \& Zajtseva, G. V. 1991, IBVS, 3680

Donati, J.-F., \& Collier Cameron, A. 1997, MNRAS, 291, 1

Dorren J. D., \& Guinan E. F. 1992, BAAS, 24, 1205

Dorren, J. D., \& Guinan, E. F. 1994, ApJ, 428, 805

Dorren, J. D., Güdel, M., \& Guinan, E. F. 1995, ApJ, 448, 431
Elstner, D., \& Korhonen, H. 2005, AN, 326, 278

Fluri, D. M., \& Berdyugina, S. V. 2004, Sol. Phys., 224, 153

Fröhlich, H.-E., Tschäpe, R., Rüdiger, G., \& Strassmeier, K. G. 2002, A\&A, 391, 659

Granzer, T., Reegen, P., \& Strassmeier, K. G. 2001, AN, 322, 325

Güdel, M., Schmitt, J. H. M. M., \& Benz, A. O. 1995a, A\&A, 302, 775

Güdel, M., Schmitt, J. H. M. M., Benz, A. O., \& Elias, N. M. II. 1995b, A\&A, 301, 201

Güdel, M., Guinan, E. F., Mewe, R., Kaastra, J. S., \& Skinner, S. L. 1997a, ApJ, 479, 416

Güdel, M., Guinan, E. F., \& Skinner, S. L. 1997b, ApJ, 483, 947

Järvinen, S. P., Berdyugina, S. V., Tuominen, I., Cutispoto, G., \& Bos, M. 2005, A\&A, 432, 657

Korhonen, H., Berdyugina, S. V., \& Tuominen, I. 2002, A\&A, 390, 179

Kövári, Zs., Strassmeier, K. G., Granzer, T., et al. 2004, A\&A, 417, 1047

Lockwood, G. W., Skiff, B. A., \& Radick, R. R. 1997, ApJ, 485, 789

Messina, S., \& Guinan, E. F. 2002, A\&A, 393, 225

Messina, S., \& Guinan, E. F. 2003, A\&A, 409, 1017

Montes, D., López-Santiago, J., Fernández-Figueroa, M. J., \& Gálvez, M. C. 2001, A\&A, 379, 976

Moss, D. 2004, MNRAS, 352, L17

Moss, D. 2005, A\&A, 432, 249

Olsen, E. H. 1983, A\&AS, 323, 151

O’Neil, D., Neff, J. E., Saar, S. H., \& Cuntz, M. 2004, AJ, 128, 1082

Press, W. H., Teukolsky, S. A., Vetterling, W. T., \& Flannery, B. P. 1992 in Numerical Recipes in FORTRAN - The Art of Scientific Computing, Second Edition (New York, USA: Cambridge University Press), 569

Radick, R. R., Lockwood, G. W., Skiff, B. A., \& Baliunas, S. L. 1998, ApJS, 118, 239

Saar, S. H., \& Bookbinder, J. A. 1998 in Proc. Tenth Cambridge Workshop on Cool Stars, Stellar Systems, and the Sun, ed. R. Donahue, \& J. Bookbinder, ASP Conf. Ser., 154, 1560

Saar, S. H., \& Brandenburg, A. 1999, ApJ, 524, 295

Scheible, M. P., \& Guinan, E. F. 1994, IBVS, 4110

Schüssler, M., Caligari, P., Ferriz-Mas, A., Solanki, S., \& Stix, M. 1996, A\&A, 314, 503

Strassmeier, K. G., Bartus, J., Cutispoto, G., \& Rodonó, M. 1997a, A\&AS, 125, 11

Strassmeier, K. G., Boyd, L. J., Epand, D. H., \& Granzer, Th. 1997b, PASP, 109, 697

Strassmeier, K. G., \& Rice, J. B. 1998, A\&A, 330, 685

Strassmeier, K. G., Serkowitsch, E., \& Granzer Th. 1999, A\&AS, 140, 29

Vaughan, A. H., \& Preston, G. W. 1980, PASP, 92, 385 